Alll02 69766?

NATL INST OF STANDARDS \& TECH R.IC

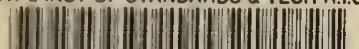

A11.0269

A11 102697667

Koepke, Galen

ac100.U56 N0.87-3068 1987 V19 C.1 NBS-P

LOG-PERIODIC DIPOLE ANTENNAS FOR GENERATING TEST FIELDS

Galen H. Koepke

David A. Hill

Mark T. Ma

National Bureau of Standards

U.S. Department of Commerce

Boulder, Colorado 80303-3328

June 1987 



\section{ANALYSIS OF AN ARRAY OF \\ LOG-PERIODIC DIPOLE ANTENNAS FOR GENERATION TEST FIELDS}

Galen H. Koepke

David A. Hill

Mark T. Ma

Electromagnetic Fields Division

Center for Electronics and Electrical Engineering

National Engineering Laboratory

National Bureau of Standards

Boulder, Colorado 80303-3328

June 1987

Sponsored in part by

Naval Surface Weapons Center

Dahlgren, Virginia 22448

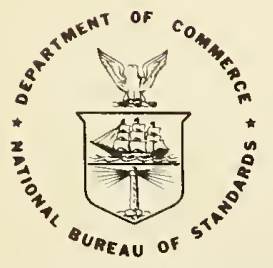

U.S. DEPARTMENT OF COMMERCE, Malcolm Baldrige, Secretary

NATIONAL BUREAU OF STANDARDS, Ernest Ambler, Director 



\section{CONTENTS}

\section{Page}

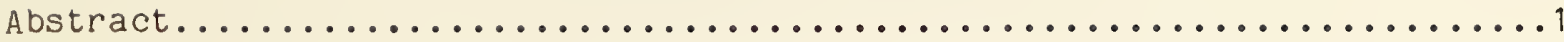

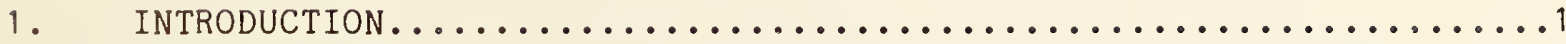

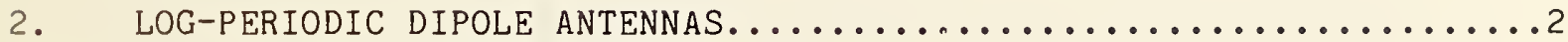

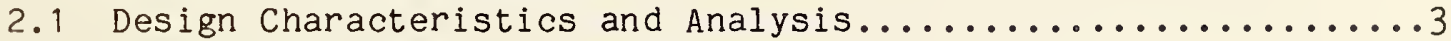

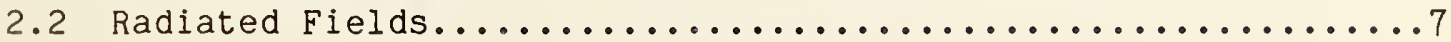

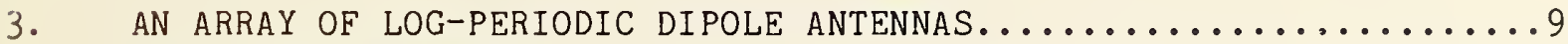

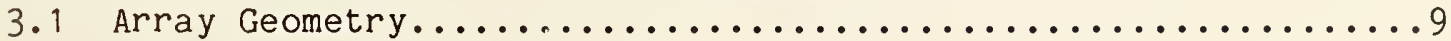

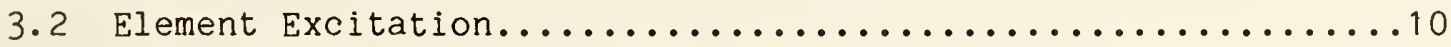

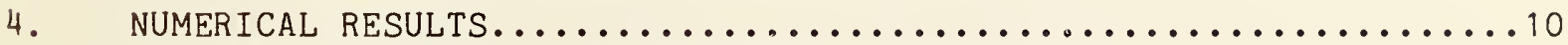

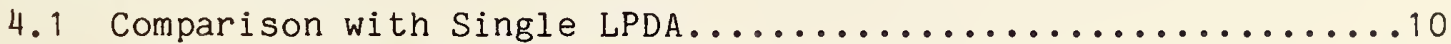

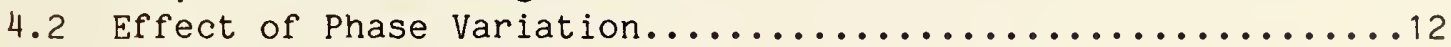

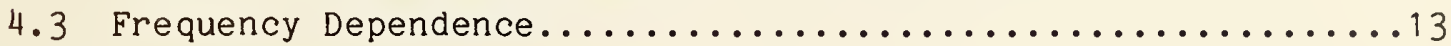

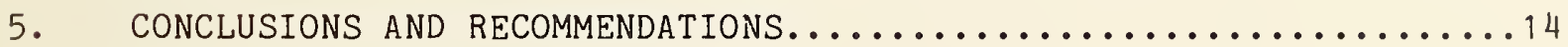

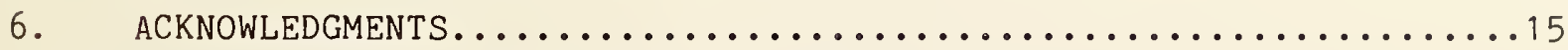

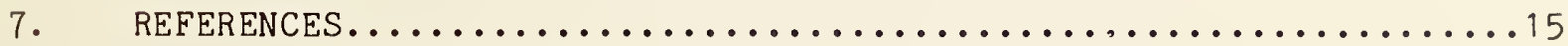





\title{
ANALYSIS OF AN ARRAY OF LOG-PERIODIC DIPOLE ANTENNAS FOR GENERATING TEST FIELDS
}

\author{
Galen H. Koepke, David A. Hill, and Mark T. Ma \\ Electromagnetic Fields Division \\ National Bureau of Standards \\ Boulder, CO 80303
}

\begin{abstract}
An analysis of log-periodic dipole antennas was extended to study their use in arrays designed for electromagnetic susceptibility measurements. Parameters of an array of $f$ ive log-periodic dipole antennas were calculated and in some cases compared to a single log-periodic dipole antenna. These parameters were used to evaluate the tradeoffs that exist in the design of an optimum transmitting antenna for susceptibility measurements.
\end{abstract}

Key words: broadband antenna; electromagnetic susceptibility; log-periodic dipole antenna; near-field phased array.

\section{INTRODUCTION}

A method has been developed to synthesize a desired field distribution over a limited volume for electromagnetic susceptibility (EMS) testing [1]. This technique uses an array of elements fed in such a manner that a given field distribution (typically a plane wave) is generated in the near-field region of the array. An array designed in this manner is known as a nearfield phased array. The technique has been verified with an array of YagiUda antennas [2] and has shown good agreement with theory and experiment. The ability to generate a given test field at a particular fixed frequency [2] is useful for verifying a concept, but a general purpose EMS facility should perform over a broad range of frequencies. There is a need for wide frequency coverage in order to perform measurements with a minimum of mechanical adjustments and equipment changes. The motivation for this study is to examine the possibilities of constructing an array of broadband elements in an effort to extend the usable bandwidth of the array and to 
determine what enhancements can be obtained to the field strength at a particular test point by using an array instead of a single broadband element.

Devices such as transverse electromagnetic (TEM) cells [3] and parallel plate transmission lines are useful for frequencies below about 50 $\mathrm{MHz}$ and compact ranges [4] for use at higher frequencies above $1 \mathrm{GHz}$ which can be used to produce TEM fields for testing purposes. There is a range of frequencies between $50 \mathrm{MHz}$ and $1 \mathrm{GHz}$ where this near-field phased array may find greatest application, and the attention will be given to that band.

A common, proven broadband antenna that radiates linear polarization and is used in this frequency range is the log-periodic dipole antenna (LPDA). This antenna design has received considerable attention $[5,6,7,8,9]$ and has been modeled theoretically. For these reasons the LPDA was chosen for the elements of the phased array. Some of the other frequencyindependent antennas [10] are not useful for our purposes because they radiate circular polarization. The TEM horn [11] is broadband and radiates linear polarization, but its field distribution is difficult to calculate.

This report is organized in the following manner. Section 2 details the analysis of the LPDA including geometry and circuit models. This section includes a description of the LPDA used for the array study and describes the important characteristics of the antenna. Section 3 details a simple array configuration and describes the calculations used to arrive at the field in front of the array. Section 4 gives the comparisons of the array of LPDAs vs a single LPDA at various operating frequencies and test points. Section 5 draws some conclusions and makes recommendations for future work.

\section{LOG-PERIODIC DIPOLE ANTENNAS}

Log-periodic dipole antennas belong to a class of antennas that exhibit nearly frequency-independent characteristics [12]. They are 
actually arrays of dipole antennas, but we refer to the array as an antenna in order to avoid confusion later when we form an array of LPDAs. The input impedance and radiation pattern of a properly designed LPDA vary periodically with the logarithm of frequency and the variation within the logarithmic period is negligible [13]. These frequency-independent properties are bandlimited by the fact that the actual antenna is a truncation of an infinite structure, but given enough properly sized and spaced elements the bandwidth is arbitrarily large.

\subsection{Design Characteristics and Analysis}

A typical log-periodic dipole antenna is shown in figure 1. The antenna is an array of parallel center-fed linear dipoles lying side by side in a plane. The lengths and radii of dipole elements form a geometric progression with a common ratio $\tau$, called the scale factor:

$$
\frac{h_{n}}{n_{n+1}}=\frac{a_{n}}{a_{n+1}}=\tau, \quad n=1,2,3, \ldots, N-1,0<\tau<1,
$$

where $h_{n}$ is the half-length, $a_{n}$ is the radius of the $n t h$ dipole, and $N$ is the total number of dipoles in the antenna. The ratio of half-length to radius is constant for all dipoles. (However, in examining several commercial antennas the radius appears to be constant over the entire antenna using the same materials for all dipoles. This analysis assumes the antenna is constructed with the radius of each dipole satisfying equation 1.) A line through the ends of the dipole elements makes an angle $\alpha$ with the array axis at the virtual apex 0 . The spacing factor $\sigma^{\prime}$ is defined as the ratio of the distance between two adjacent elements to twice the length of the larger element and is constant for a given LPDA. The geometry of figure 1 relates $\sigma^{\prime}$ to $\tau$ and $\alpha$ :

$$
\sigma^{\prime}=\frac{d_{n}}{4 h_{n+1}}=\frac{y_{n+1}-y_{n}}{4 h_{n+1}}=\frac{\left(h_{n+1}-h_{n}\right) \cot \alpha}{4 h_{n+1}}=\frac{1}{4}(1-\tau) \cot \alpha .
$$


The following analysis is drawn from Ma [8] and is based on the work of Carrel [7]. The calculations treat the LPDA as a parallel circuit combination of two distinct parts, the feeder circuit which is a balanced constant-impedance feed line and the dipole antennas which are connected such that the phase reverses for adjacent dipoles. The voltages and currents at the input terminals of the dipole elements are determined by solving circuit equations. A schematic representation of the circuit elements is shown in figure 2. The current-voltage relations for the dipole element circuit (figure $2 a$ ) can be written as

$$
\left[\mathrm{V}_{\mathrm{a}}\right]=\left[\mathrm{Z}_{\mathrm{a}}\right]\left[\mathrm{I}_{\mathrm{a}}\right] \text { or } \quad\left[\mathrm{I}_{\mathrm{a}}\right]=\left[\mathrm{Z}_{\mathrm{a}}\right]^{-1}\left[\mathrm{~V}_{\mathrm{a}}\right]
$$

where

$$
\left[I_{\mathrm{a}}\right]=\left[\begin{array}{c}
\mathrm{I}_{1 \mathrm{a}} \\
\mathrm{I}_{2 \mathrm{a}} \\
\vdots \\
\mathrm{I}_{\mathrm{Na}}
\end{array}\right] \quad \text { and } \quad\left[\mathrm{V}_{\mathrm{a}}\right]=\left[\begin{array}{c}
\mathrm{V}_{1 \mathrm{a}} \\
\mathrm{V}_{2 \mathrm{a}} \\
\vdots \\
\mathrm{V}_{\mathrm{Na}}
\end{array}\right]
$$

are $\mathrm{Nx} 1$ column matrices representing, respectively, the driving base currents and response voltages for the dipole elements, and

$$
\left[z_{a}\right]=\left[\begin{array}{lrlr}
z_{11 a} & z_{12 a} & \cdots & z_{1 N a} \\
z_{21 a} & z_{22 a} & \cdots & z_{2 N a} \\
\ldots \ldots & \ldots & \ldots & \ldots \\
z_{N 1 a} & z_{N 2 a} & \cdots & z_{N N a}
\end{array}\right]
$$

is the associated $\mathrm{NxN}$ open-circuit impedance matrix.

The main diagonal of $\left[z_{a}\right]$ in (5) represents the self-impedances of the dipoles while the off-diagonal elements represent the mutual impedances between dipoles indicated by the indices. All of these impedances can be 
calculated (Ma [8], eq 4.32 or eq 4.33 and eq 4.38 or eq 4.44). These impedance calculations are derived by the induced-emf method with the King and wu [14] three-term assumption for the current distribution on the dipole antennas.

The current-voltage relations for the feeder circuit shown in figure $2 \mathrm{~b}$ can be expressed by

$$
\left[I_{f}\right]=\left[Y_{f}\right]\left[V_{f}\right]=\left[Y_{f}\right]\left[V_{a}\right]
$$

where

$$
\left[I_{f}\right]=\left[\begin{array}{c}
I_{1 f} \\
I_{2 f} \\
\vdots \\
I_{N f}
\end{array}\right] \quad \text { and } \quad\left[v_{f}\right]=\left[\begin{array}{c}
V_{1 f} \\
V_{2 f} \\
\vdots \\
V_{N f}
\end{array}\right]=\left[\begin{array}{c}
V_{1 a} \\
V_{2 a} \\
\vdots \\
V_{N a}
\end{array}\right]
$$

are the driving currents and response voltages, respectively, for each section of the transmission line which constitutes a complete feeder circuit, and

$$
\left[Y_{f}\right]=\left[\begin{array}{cccc}
Y_{11 f} & Y_{12 f} & \cdots & Y_{1 N f} \\
Y_{21 f} & Y_{22 f} & \ldots & Y_{2 N f} \\
Y_{N 1 f} & Y_{N 2 f} & \ldots & Y_{N N f}
\end{array}\right]
$$

is the associated $\mathrm{NxN}$ short-circuit admittance matrix of the feeder. The matrix elements of $\left[\mathrm{Y}_{\mathrm{f}}\right]$ in (8) depend on the lengths of the transmission line in each section and the characteristic admittance $Y_{O}$, which is known for a given transmission line. The elements of $\left[\mathrm{Y}_{\mathrm{f}}\right]$ can be calculated by (Ma [8] eq 5.9). 
The feeder and antenna circuits are connected in parallel at the dipole bases. Hence there is no difference between the feeder voltage matrix $\left[\mathrm{V}_{\mathrm{f}}\right]$ and the antenna voltage matrix $\left[\mathrm{V}_{\mathrm{a}}\right]$, and [V] is used to represent both, as shown in figure $2 \mathrm{c}$.

Adding (3) and (6) we obtain the total input current matrix:

$$
[I]=\left[I_{a}\right]+\left[I_{f}\right]=\left\{[U]+\left[Y_{f}\right]\left[Z_{a}\right]\right\}\left[I_{a}\right]
$$

where [U] is the $\mathrm{N} \times \mathrm{N}$ unit matrix.

The matrix elements of [I] represent the input currents to each node point (dipole base) at the point where the antenna and feeder circuits are connected. The dipole base current matrix $\left[\mathrm{I}_{a}\right]$ can be determined from (9) by matrix inversion:

$$
\left[I_{a}\right]=\left\{[U]+\left[Y_{f}\right]\left[Z_{a}\right]\right\}^{-1}[I]
$$

where the elements of [I] are all zero except for the driving current to the array at the base of the smallest dipole, which is assumed to be $1 \mathrm{~A}$.

The response voltages appearing at the dipole bases can then be determined by substituting (10) into (3). The input impedance $z_{i n}$ of the entire array, which is numerically equal to the voltage across the feed point since the input current is assumed to be 1 , is

$$
Z_{\text {in }}=V_{1}=\sum_{i=1}^{N} Z_{1 i a} I_{i a}=R_{i n}+j X_{i n}
$$

Figure 3 shows the details of an 18-element LPDA for use in the 200$600 \mathrm{MHz}$ frequency range. This antenna is given as an example in ([9], example 6.3, pg. 299) and has characteristics that are of interest in this 
study. The base currents of the dipole elements at $300 \mathrm{MHz}$ calculated by the above method are shown in figure 4, and comparisons are made with moment method calculations by stutzman and Thiele [9] for a transmission line impedance of $83 \Omega$ as given in [9] (this impedance is changed to a more common value of $50 \Omega$ for all subsequent calculations). The data in figure 4 show the relative base currents for each dipole in the antenna and illustrate the three different regions of the LPDA [7]. The first region is called the transmission region which includes the feed point (dipole 1) and the first few elements that have very low base currents. The dipoles in this region have the smallest physical dimensions and define the upper frequency limit of the antenna. The second region is referred to as the active region. Here the dipoles have relatively high base currents, and this is where the majority of radiation takes place. The dipoles in this region have lengths close to one-half wavelength. The remaining longer dipoles are in the unexcited region. This region defines the lower frequency limit of the antenna.

\subsection{Radiated Fields}

The radiated fields of the antenna can be calculated using assumed current distributions on the dipoles, the relative amplitudes of the base current for each dipole, and the geometry of the array. The components of the far-field can be evaluated in closed form ([8], section 5.2) for the three-term current. However, for this application the region of interest includes close distances in front of the array. The integrals for terms that include the higher inverse powers of distance, which become more significant at close distances, cannot be easily evaluated analytically and a numerical integration becomes necessary.

The formulation for the fields radiated by any segment of a particular dipole can be derived in terms of the vector potential [15, 16, 17]. The electric and magnetic field components are found by performing the required mathematical operations on the vector potential function. The rectangular cartesian coordinate system is chosen with the dipole orientated along the 
$x$-axis. The vector potential A at a field point ( $x, y, z)$ for this configuration can be expressed as

$$
A=a_{x} \frac{\mu}{4 \pi} \int_{-h}^{h} I\left(x^{\prime}\right) \frac{e^{-j k r}}{r} d x^{\prime},
$$

where $a_{x}$ is the unit vector in the $x$ direction, $r^{2}=\left(x-x^{1}\right)^{2}+y^{2}+z^{2}$, and $I\left(x^{\prime}\right)$ is the current distribution along the antenna. The electric field (E) and the magnetic field $(H)$ are related to $A$ by

$$
E=-j \omega A-\frac{j}{\omega \mu \varepsilon} \quad \nabla(\nabla \cdot A) \quad \text { and } \quad H=\frac{1}{\mu} \quad(\nabla \times A) \text {, }
$$

where $\omega$ is the radian frequency, with $e^{j \omega t}$ as the time dependency, $\mu$ is the permeability, and $\varepsilon$ is the permittivity of the transmission medium (free space). The indicated derivatives are performed retaining all the higher inverse powers of $r$. The resulting integrals are then evaluated numerically with Simpson's $1 / 3$ rule [18] using the current weightings derived for each dipole. The fields from each dipole in the LPDA are summed together at a given test point to give the total field from the array of dipoles.

A sample calculation for the electric field along the boresite (vs -y at $x=z=0$ ) of the LPDA using the asymptotic expression for the far-field and the complete expressions containing the near-field terms evaluated via a numerical integration routine is shown in figure 5. These data are useful for comparing the two calculations and verifying the near-field derivation and integration routines. The data also illustrate the region where farfield calculations are no longer adequate and produce incorrect results. The remaining calculations utilize the near-field terms and should be valid at all distances. 


\section{AN ARRAY OF LOG-PERIODIC DIPOLE ANTENNAS}

The single LPDA with its endfire radiation pattern and fixed geometry does not provide means to vary the field structure. A desired flexibility can be gained by constructing an array of LPDAs with the amplitude and phase of the feed current, number of elements, and the array geometry providing the necessary parameters required to control the radiated field. These parameters are somewhat arbitrary yet interrelated. The intended use of the array will, in most cases, determine practical choices for the array parameters. This application requires the array to be easily handled, thus limiting the overall size; to provide a maximum field strength for a given input power; and to provide acceptable field uniformity over the test volume. After considering the array geometry, the maximum field strength vs field uniformity compromises will be discussed.

\subsection{Array Geometry}

The array geometry shown in figure 6 details a planar five-element broadside array of LPDAS. This configuration is a minimum sized array that will provide some flexibility in the control of the field structure in a small test volume. Additional elements would increase this capability but at the expense of feed complexity and ease of handling. The five elements are arranged with the outer four on the corners of a square and one in the center. The symmetry of this geometry permits the simplest possible feed network with the center element as one feed value and the outer four elements taking another value. The need to illuminate a volume of cubic or spherical proportions has ruled out other possible geometries such as arranging the elements in a linear fashion. The elements are spaced sufficiently far apart (on the order of a wavelength) such that effects from mutual coupling between the LPDA elements can be neglected. Mutual coupling was found to be negligible in an earlier near-field array [2], and there are no corrections for these effects in this analysis. 


\subsection{Element Excitation}

As mentioned above, the five-element array has only two values of the feed current, with the outer four elements requiring an identical value. The near-field synthesis technique can be used to determine what the feed should be for a given field structure. Typical improvements in field uniformity for a similar array can be found in [2] and a similar analysis for this array is not repeated here. It should be noted that the near-field synthesis technique tends to prescribe feed values that are not well matched to the common $1 / 2-1 / 2$ power splitters [2]. Attenuators are then required increasing the losses in the feed system. For an application where field uniformity or prescribed structure is the most important feature, these additional losses can easily be tolerated or a feed system specificically designed based on these requirements. However, for the purpose of this study emphasizing the frequency dependent nature of the array along with the potential application requiring the maximum field strength for a given input power for which attenuators in the feed system should be avoided, the amplitude weightings are chosen such that common power splitters may be used. The array elements are then assumed to be fed with equal splitting where the center element receives $1 / 2$ and the outer four elements each receive $1 / 8$ of the power. The relative phase is adjustable and will be examined. The phase can be determined by hot-spot theory [19] which results in the outer elements leading the center such that the field is in phase at the test point. Alternatively, the phase of the outer elements can be retarded relative to the center to produce a more uniform field over a wider area. The actual phase chosen for the array will be determined by the application.

4. NUMERICAL RESULTS

4. 1 Comparison with Single LPDA

The intended use for an array of LPDAs is to produce a specified field environment for EMS testing. A typical measurement involves positioning the 
equipment under test (EUT) and the transmitting antenna such that the EUT is exposed to the specified field to be generated by the transmitting antenna. In many situations the EUT may be quite large (e.g.,. aircraft) and it is more practical to move the transmitting antenna rather than the EUT to provide various illuminations for the EUT. A single LPDA is obviously more convenient than an array of several LPDAs and in some situations may be a good choice.

Many susceptibility tests require high field strengths to simulate real environments that exist where the equipment is to be used. Higher field levels obviously require larger power amplifiers, but some enhancement may also be attained by reducing the distance between the transmitting antenna and the EUT. Figure 7 details what happens to the ratio of the dominant far-field components $\left(\mathrm{E}_{\mathrm{x}} / \mathrm{H}_{\mathrm{z}}\right)$ as this distance is varied. The $\mathrm{E}_{\mathrm{x}} / \mathrm{H}_{\mathrm{z}}$ ratio begins to deviate from the free space impedance value of $120 \pi \Omega$ at about 2 wavelengths separation distance for both the single LPDA and the array of 5 LPDAs whose individual LPDAs are fed in-phase. This deviation from $120 \pi \Omega$ indicates the deterioration of the plane wave field structure into a complex near-field environment. Figure 7 indicates that either antenna may be usable to a fraction of a wavelength with relatively minor deviation of the wave impedance. However, it is not practical to operate an antenna at such close distances to a typically reflective EUT because of the interaction and mutual coupling that will occur between the antenna and the EUT.

The array has some advantages in terms of field strength in the test zone, control over field uniformity, and flexibility in the size of the test zone. The enhancement in field strength is demonstrated in figure 8 for the case where the array elements are fed in phase. The dominant electric field component $\left(E_{x}\right)$ is calculated for a single LPDA and for the array of 5 LPDAs along the boresite of each. The array is able to concentrate more energy in the test zone for the same total input power than a single LPDA. The improvement in field strength can be seen as a function of distance in figure 9 where the ratio of $E_{x}$ from the array to $E_{x}$ produced by the single 
antenna is shown. An approximate theoretical maximum for the far-field gain of the array over that of the single LPDA is $10 \log \mathrm{N}=7 \mathrm{~dB}$. The far-field result in figure 9 is within half of one decibel of this maximum.

A comparison of the electric field patterns perpendicular to the propagation direction is shown in figures 10 and 11, here the array is fed in an in-phase manner with the same input power as the single LPDA. The ability to vary the test volume by changing the phase of the array feed system will be discussed in section 4.2. The single LPDA has no provisions for changes in the radiation pattern that could alter the field in the test zone.

4.2 Effect of Phase Variation

The effects of changing the phase of the feed system as discussed in section 3.2 can be seen in figures 12 and 13 . The data in figure 12 show the transverse amplitude variation in $E_{x}$ parallel to the LPDAs at a distance of 3 meters in front of the array for differing phase of the outer four elements with respect to the center element. The highest field strength at the peak of the beam is obtained when the outer elements have a leading phase angle. This angle can be determined by requiring all contributions at a particular test point to add in phase. The most uniform field can be seen near the phase value of -99.7 degrees. The test area of \pm 1 meter for this case is not as uniform as the single LPDA of figure 10. However, if the test area is extended to \pm 3 meters the array shows substantial improvement over the single LPDA at the perimeter. The array would show improved field uniformity in both the transverse direction shown here and in the longitudinal direction (figure 5) if the amplitude and phase of the element weightings were determined by the synthesis technique [2]. The phase angle for $E_{X}$ at corresponding locations is shown in figure 13. Similar data for the H-plane pattern are shown in figures 14 and 15. These curves demonstrate the tradeoffs that exist between maximizing power density and generating a uniform field in the test zone. 
The characteristics of the array change as a function of frequency. The assumption here is that the mechanical dimensions and connections are not altered as the frequency changes. The electrical dimensions of the array obviously then depend on frequency; the spacing of elements in terms of wavelengths is less at low frequencies and greater at high frequencies, and in the case of log-periodic elements, the actual point of maximum radiation will move along the length of the LPDA. The array cannot therefore be labeled as broadband in the same sense as the LPDA. The useful range of frequencies over which the array may be used is limited by how much variation can be tolerated.

The input impedance is one parameter that does not change dramatically with frequency as it is more a function of the element characteristics than of the array. The data shown in figure 16 are for a single 18 element LPDA over the band of 200-600 $\mathrm{MHz}$. This input impedance will become part of the array feed network which can be designed to avoid frequency dependent behavior.

The relative distribution of base currents within a single LPDA is shown in figure 17 at several frequencies. The active region of the LPDA clearly moves along the length of the LPDA. This active region radiates the majority of power so the effect here is one of varying the separation distance of the array to the EUT. This would change the amplitude of the signal but would not, in itself, change the field structure except at very close distances.

The parameter that does have a pronounced effect upon the field structure, given a fixed feed system, is the spacing of the individual LPDA array elements. The array in this example is designed with a one-wavelength spacing at the middle frequency ( $400 \mathrm{MHz})$. This translates to 0.5 wavelength at $200 \mathrm{MHz}$ and 1.5 wavelength at $600 \mathrm{MHz}$. The effects on the field structure are shown in figures 18 and 19. Again the data are for a separation of 3 meters between the antenna origin and the test plane. 


\section{CONCLUSIONS AND RECOMMENDATIONS}

An analysis of log-periodic dipole antennas was extended to study their use in arrays designed for electromagnetic susceptibility measurements. The near-field contributions to the radiated field components were included to allow the test volume to be in close proximity of the array. The computer code developed was then used to study an array of five LPDAS. The characteristics of the array were compared to a single LPDA in terms of wave impedance of the radiated fields vs distance, field strength in the test zone, field uniformity, and size of the test zone. The radiation pattern of the array for various feed phasings was examined to illustrate the compromises between field strength and field uniformity. These calculations were repeated using the in-phase case for three frequencies to show the variations that an array of fixed geometry would experience as the operating frequency is changed. The array characteristics will change with frequency and the useful bandwidth is determined by the tolerances allowed. A possible alternative to the bandwidth limitation is to adjust the mechanical spacing of the broadband elements to compensate for the change in frequency.

This information is needed to evaluate the tradeoffs that exist in the design of an optimum transmitting antenna for susceptibility measurements. An array of broadband elements can increase the field strength in the test zone, or broaden the area of uniform illumination, as compared to a single element. The advantages of the array solution must be weighed against the increased complexity and bulk of the array. This analysis is limited to the relatively simple case of a five-element array due to size and handling considerations. The flexibility provided by an array would increase as the number of elements is increased, but for the case of EMS testing the disadvantages of increased size make a large array less practical.

Some of the more sophisticated features of the near-field phased array method, synthesizing the field structure, scanning the test volume, and constraining the fields external to the test volume are not addressed in this report. Near-field synthesis techniques tend to prescribe element 
weightings of odd values [2]. The goal here was to examine an array fed in an efficient manner without additional attenuation of the driving signal in the feed network in order to maximize the energy delivered to the test zone. We recommend designing a five-element array of LPDAs and testing the array over a wide bandwidth (perhaps an octave). Measurements of the input impedance and the near-field distribution would be useful in testing the validity of neglecting mutual coupling. Further theoretical calculations of other array geometries would also be interesting. Nonuniform spacings of the array elements [8] might be helpful in increasing the bandwidth of the array.

\section{ACKNOWLEDGMENTS}

The authors are grateful to L. C. Walters for the use of the computer code that provided the core of calculations for the LPDA evaluation and saved untold hours in implementing the analysis used in this report, and to $J$. Bean and the staff of the Naval Surface Weapons Center, Dahlgren, Virginia, for their interest and support of this work. We also thank C.K.S. Miller for his continued interest and support.

\section{REFERENCES}

[1] Hill, D.A. Theory of near-field phased arrays for electromagnetic susceptibility testing. Nat. Bur. Stand. (U.S.) Tech. Note 1072; 1984 .

[2] Hill, D.A.; Koepke, G.H. A near-field array of Yagi-Uda antenna for electromagnetic susceptibility testing. Nat. Bur. Stand. (U.S.) Tech. Note $1082 ; 1985$.

[3] Crawford, M.L. Generation of standard EM fields using TEM transmission cells. IEEE Trans. Electromagn. Compat. EMC-16: 189-195; 1984.

[4] Johnson, R. C.; Ecker, H. A.; Moore, R. A. Compact range techniques and measurements. IEEE Trans. Antennas Propag. AP-17: 568-575; 1969.

[5] Isbel, D. E. Log-periodic dipole arrays. IRE Trans. Antennas and Propagation. AP-8(3): 260-267; 1960 May. 
[6] Carrel, R. L. The design of log-periodic dipole antennas, IRE National Convention Record, part 1. Vol. 9: 61-75; 1961.

[7] Carrel, R. L. Analysis and design of the log-periodic dipole antenna. University of Illinois Technical Report No. 52; Urbania, IL. 1961.

[8] Ma, M. T. Theory and application of antenna arrays. New York, NY: John Wiley \& Sons; 1974.

[9] Stutzman, W. L.; Thiele, G. A. Antenna theory and design, New York, NY: John Wiley \& Sons; 1981.

[10] Rumsey, V. H. Frequency independent antennas. New York, NY: Academic Press; 1966.

[11] Kanda, M. The effects of resistive loading of "TEM" horns, IEEE Trans. on EMC. EMC-24(2); 1982 May.

[12] Rumsey, V. H. Frequency-independent antennas, IRE National Convention Record, part 1. Vol. 5: 114-118; 1957.

[13] DuHamel, R. H.; Isbel, D. E. Broadband log-periodic antenna structures, IRE National Convention Record, part 1. Vol. 5: 119-128; 1957.

[14] King, R. W. P.; Wu, T. T. Currents, charges, and near fields of cylindrical antennas. Radio Sci. Vol. 69D(3): 429-446; 1965 March.

[15] Johnk, C. T. A. Engineering electromagnetic fields and waves. New York, NY: John Wiley \& Sons; 1975.

[16] Jordan E. C.; Balmain, K. G. Electromagnetic waves and radiating systems, second edition. Englewood Cliffs, N.J: Prentice-Hall, Inc.; 1968.

[17] Harrington, R. F. Time-harmonic electromagnetic fields. New York, NY: McGraw-Hill; 1961.

[18] LaFara, R. L. Computer methods for science and engineering. Rochelle Park, N.J: Hayden Book Company, Inc.; 1973.

[19] Gee, W.; Shung-wu Lee; Bong, N. K.; Cain, C. A.; Mittra, R.; Magin, R. L. Focused array hyperthermia applicator: theory and experiment. IEEE Trans. on Biomedical Engr. BME-31(1); 1984 January. 


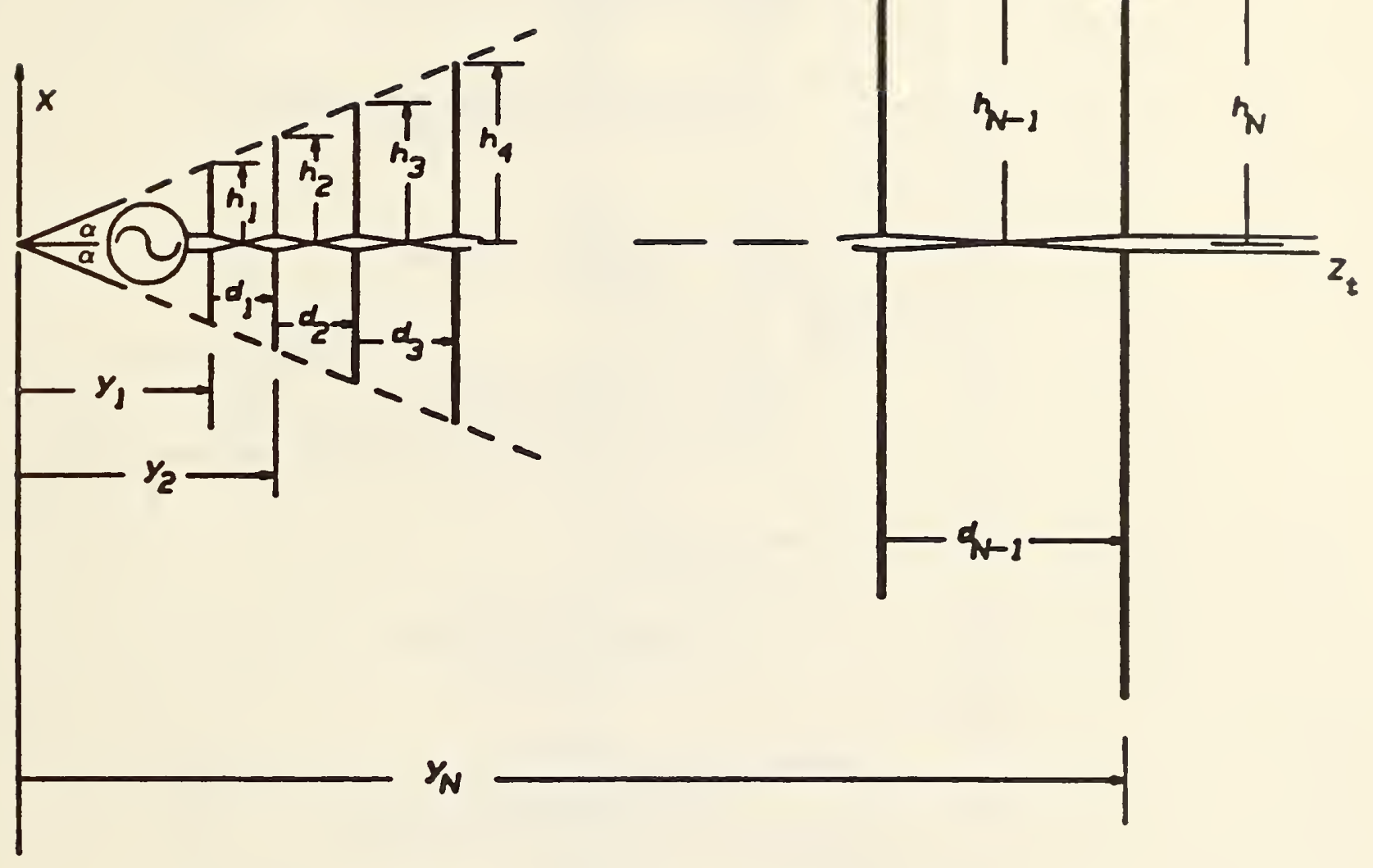

Figure 1. Geometry of a log-periodic dipole antenna. 


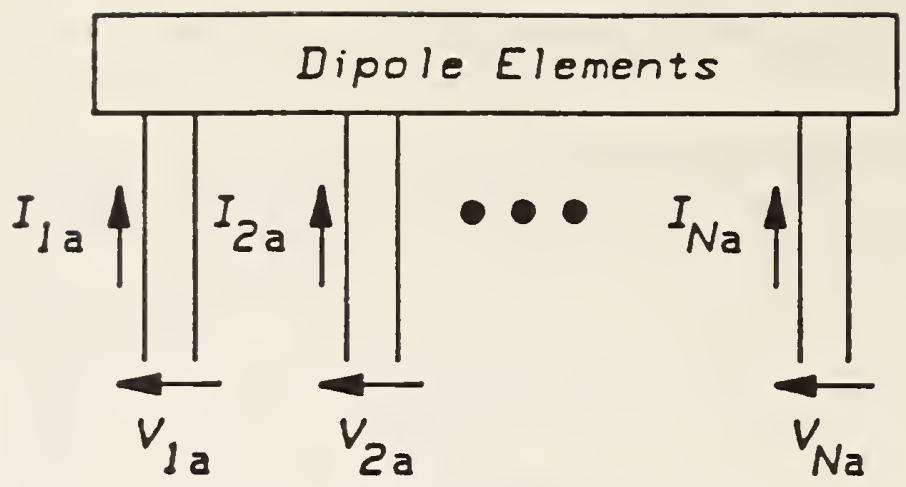

a) Dipole element circuit

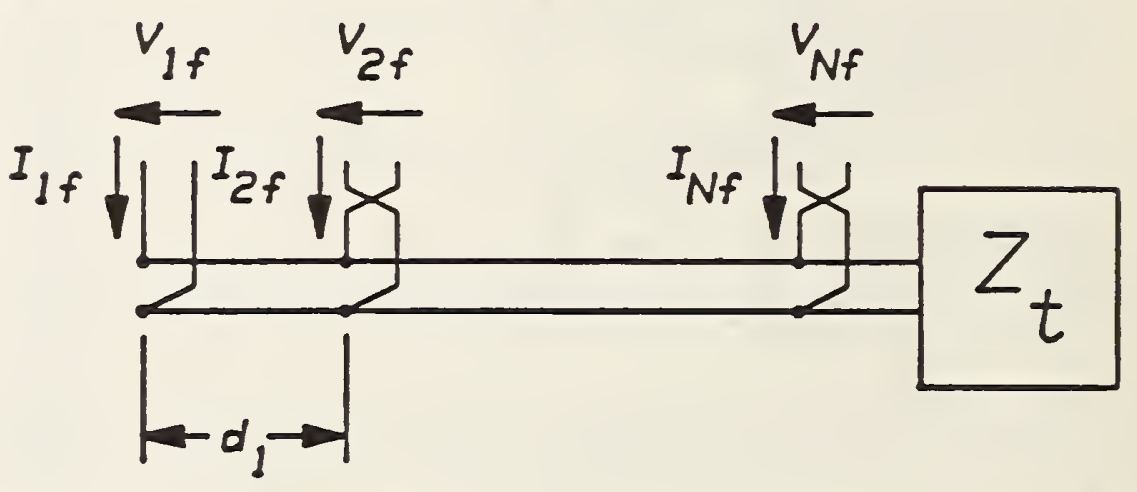

b) Feeder circuit

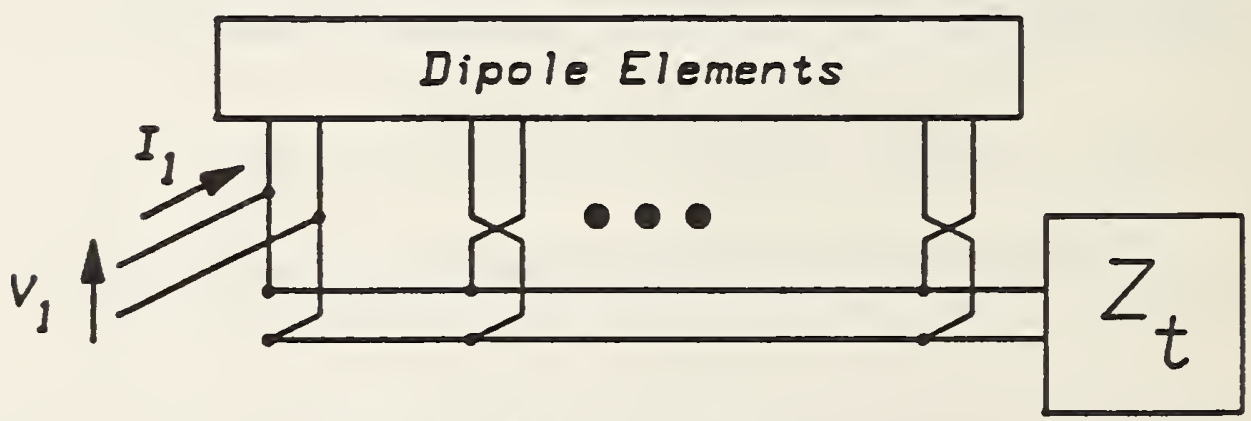

c) Complete circuit

Figure 2. Circuit model of a log-periodic dipole antenna. 


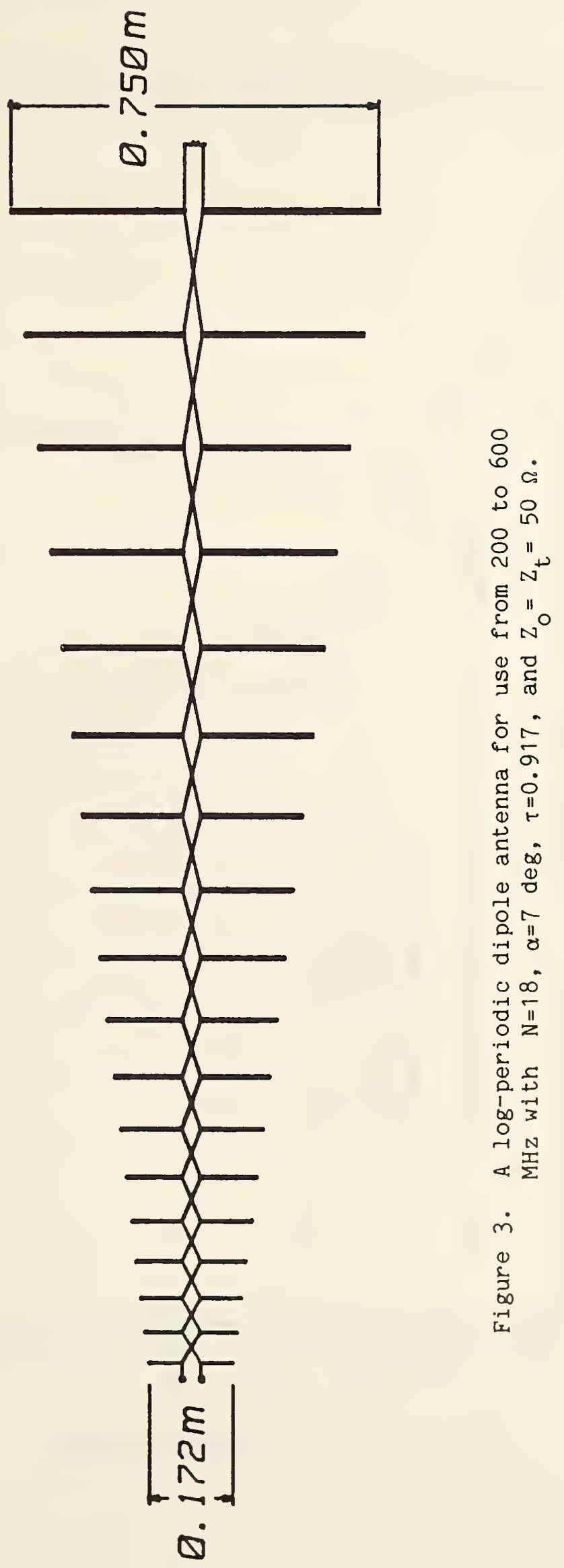




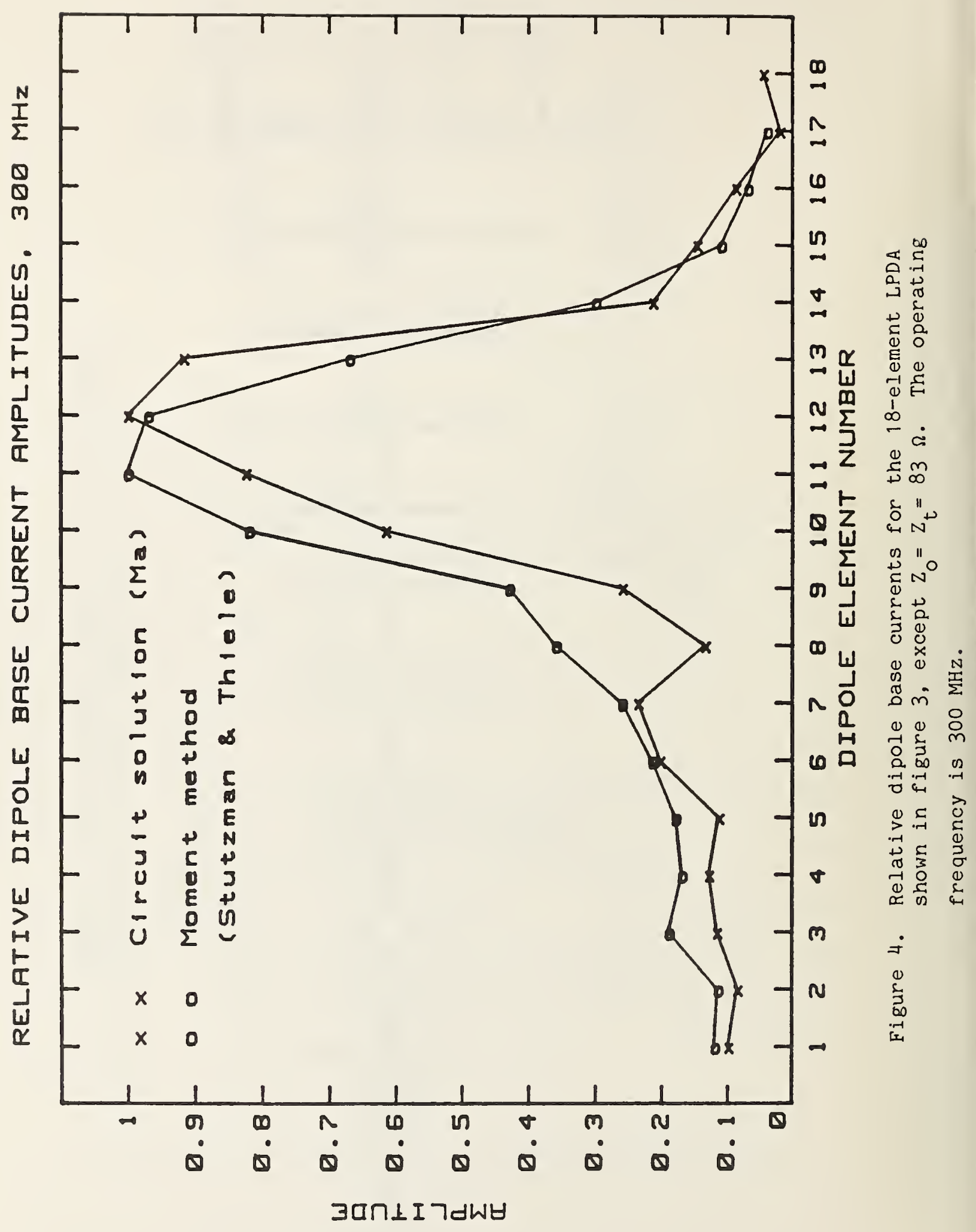




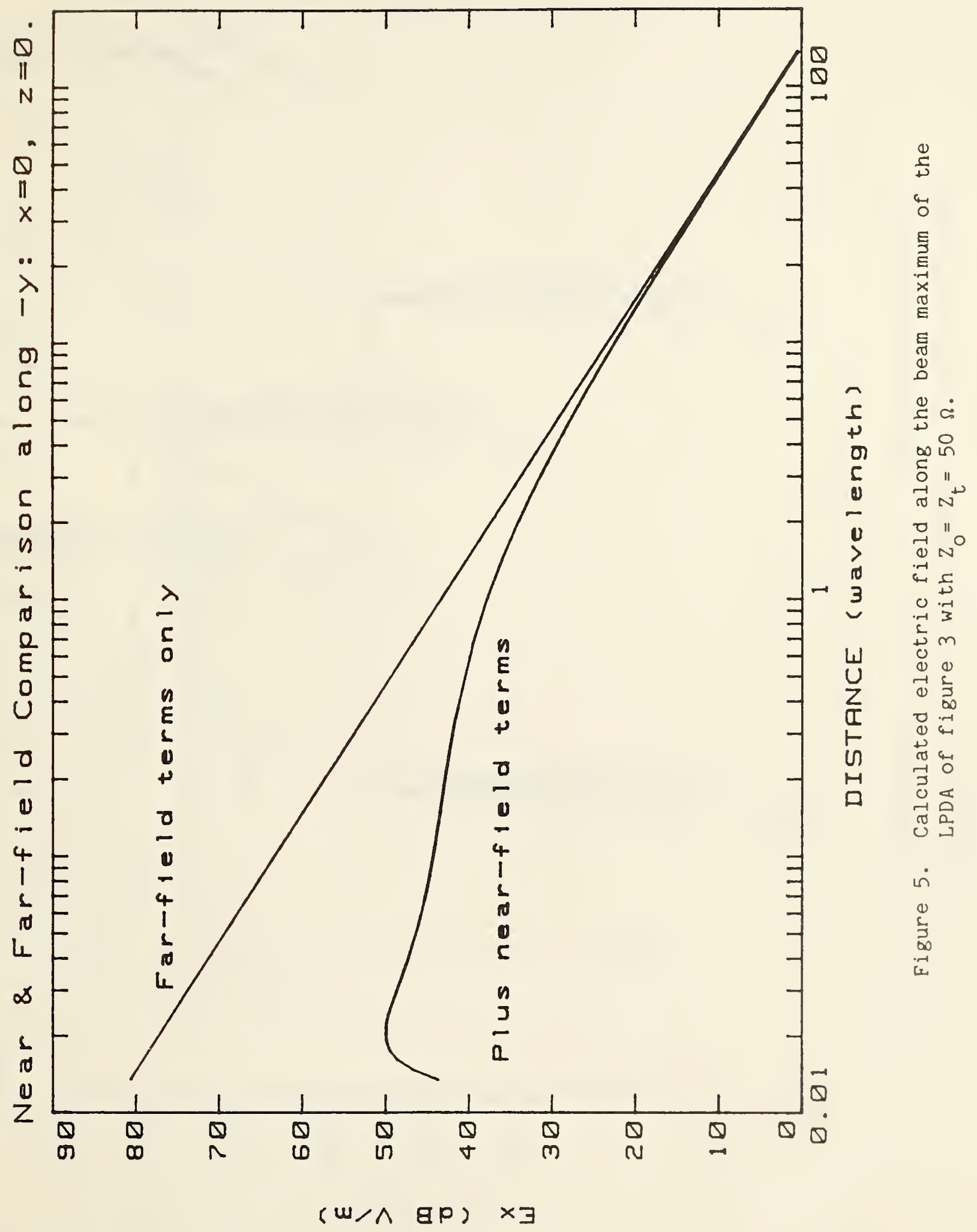




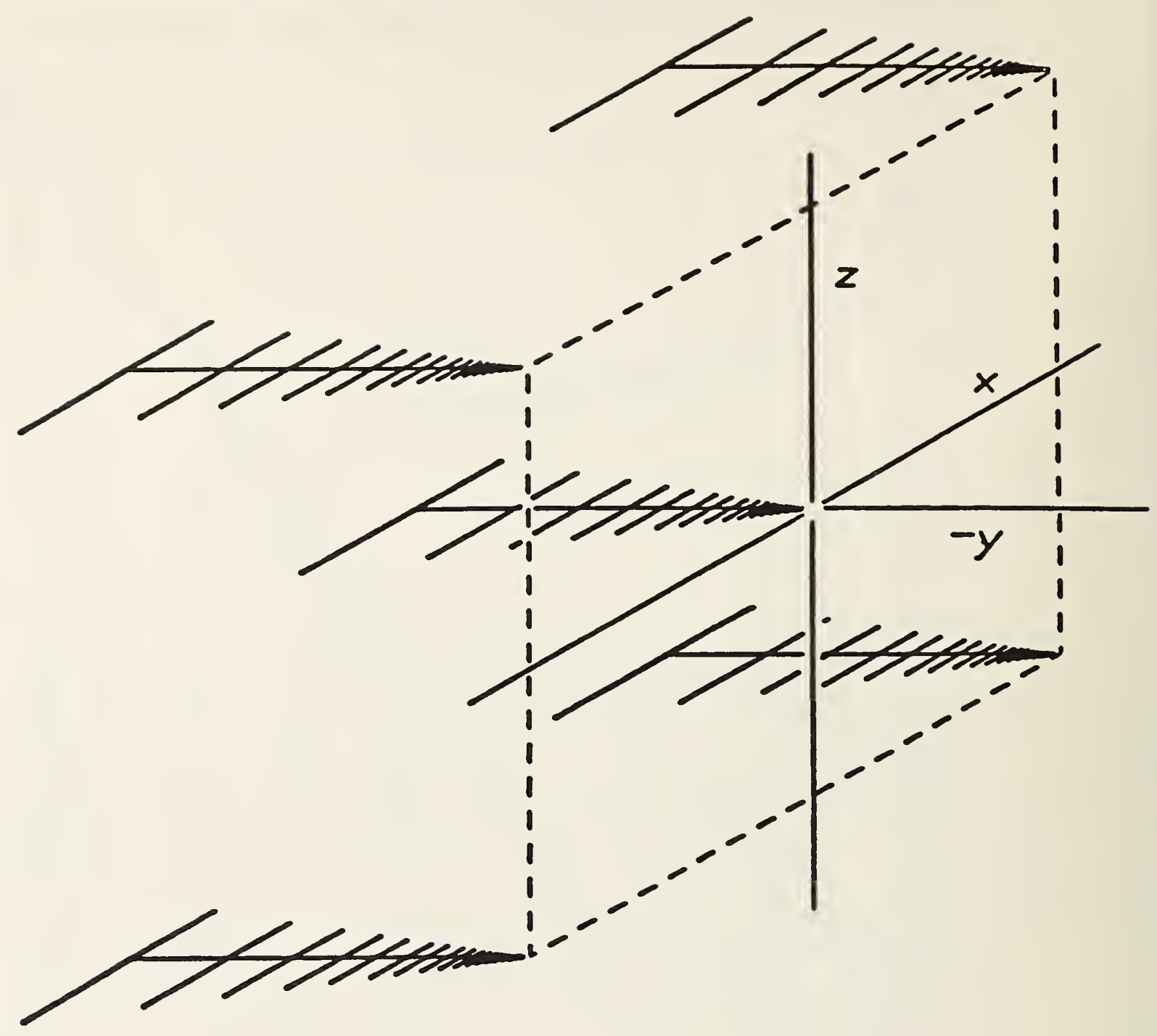

Figure 6. A five-element array of LPDAs. 


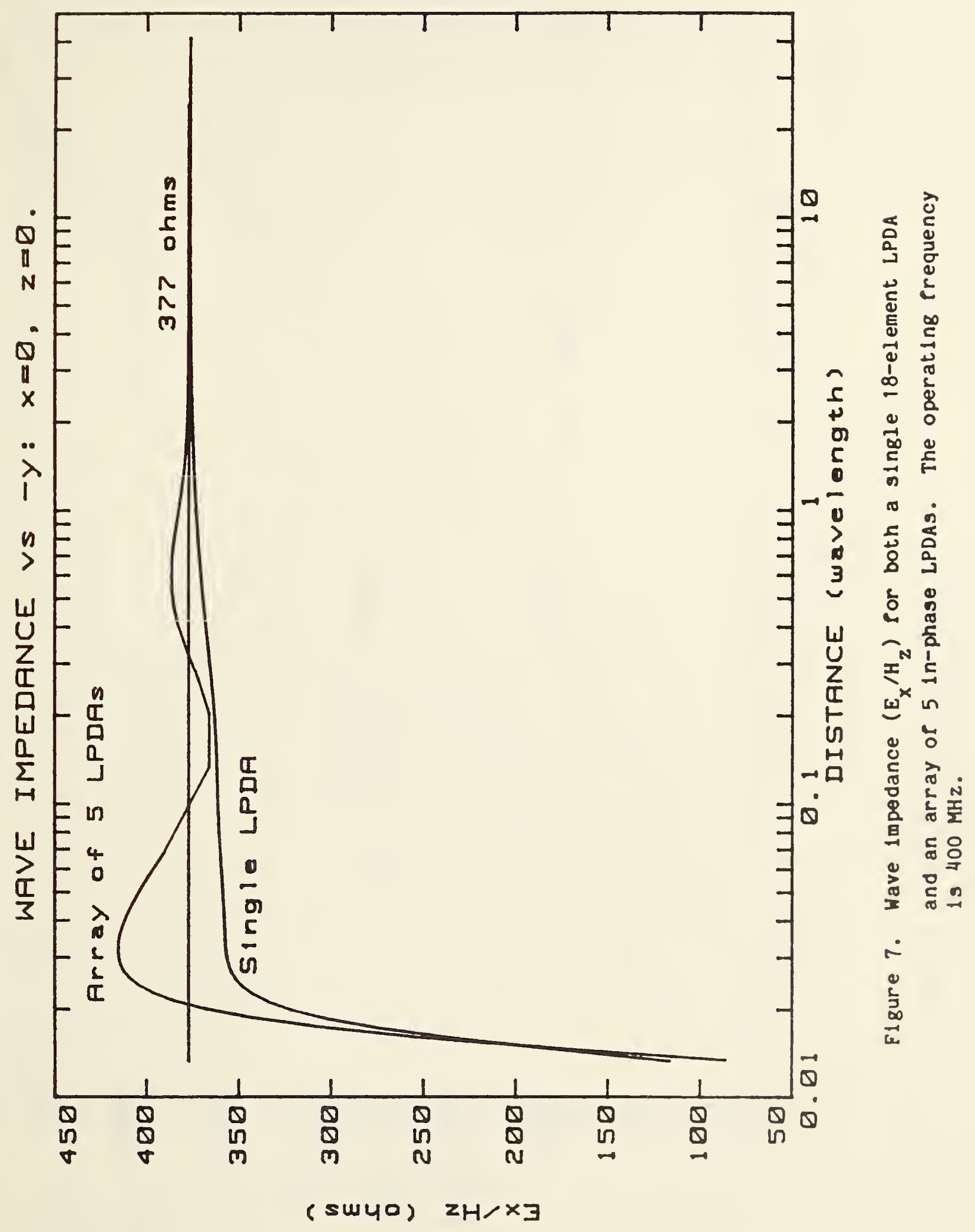




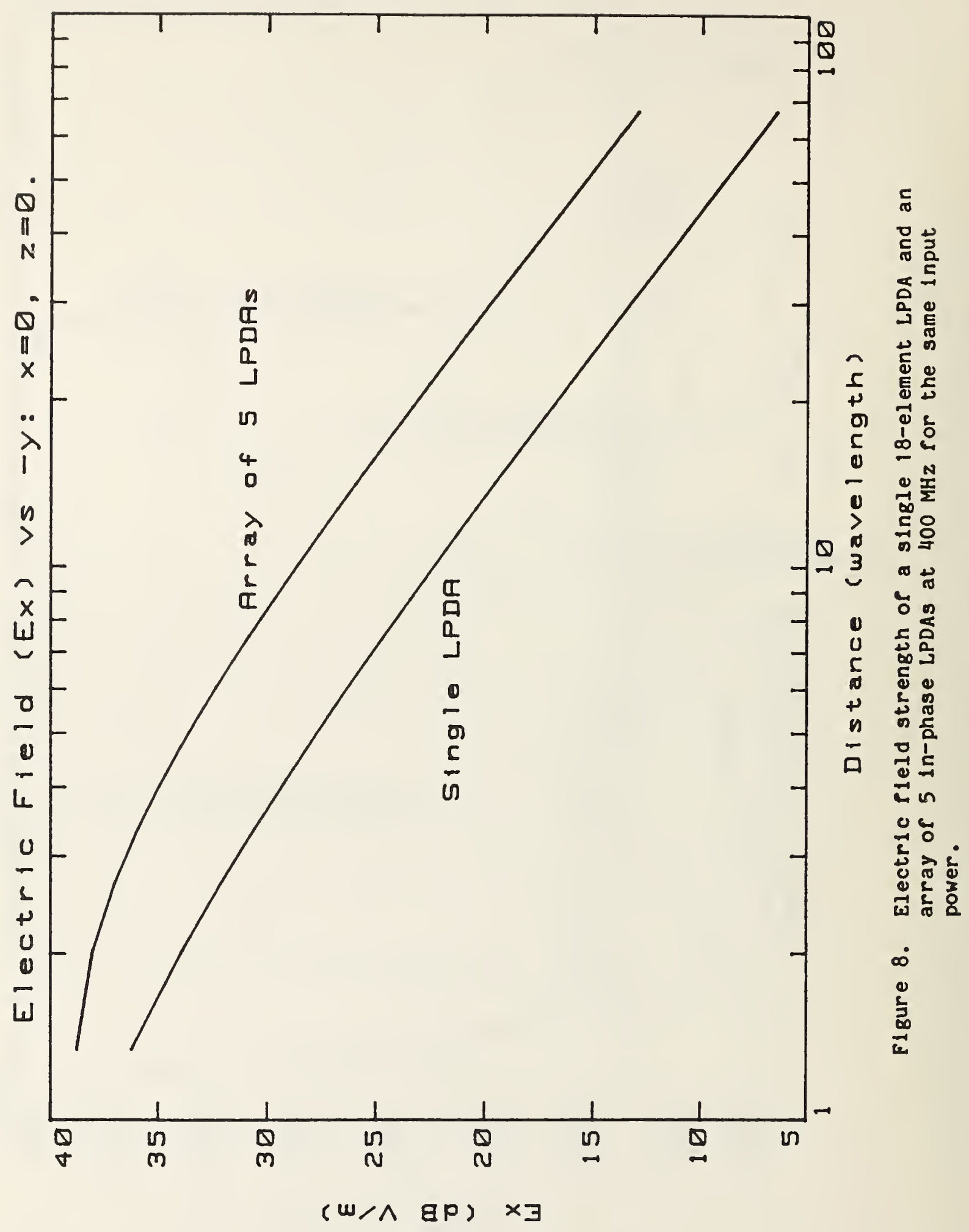




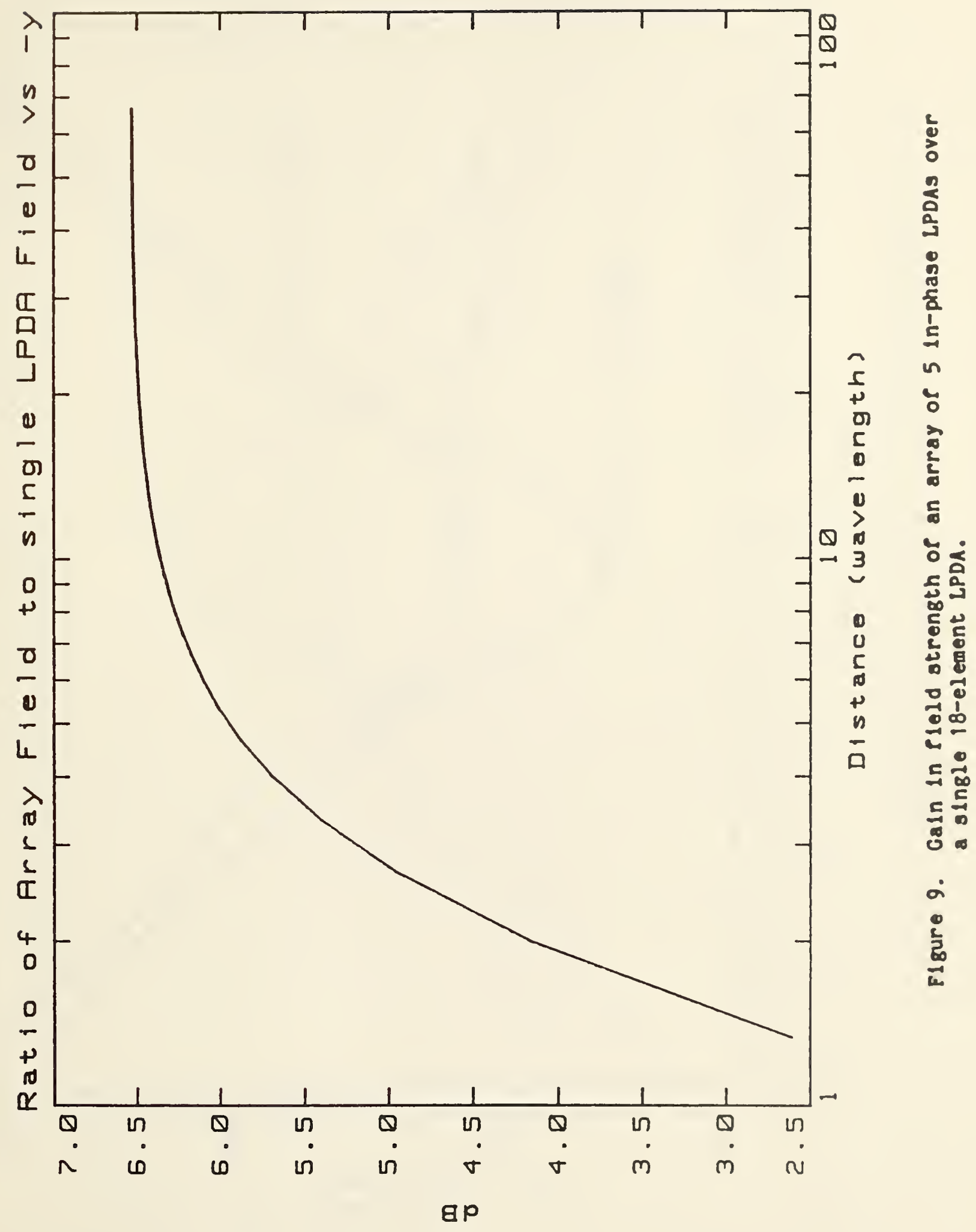




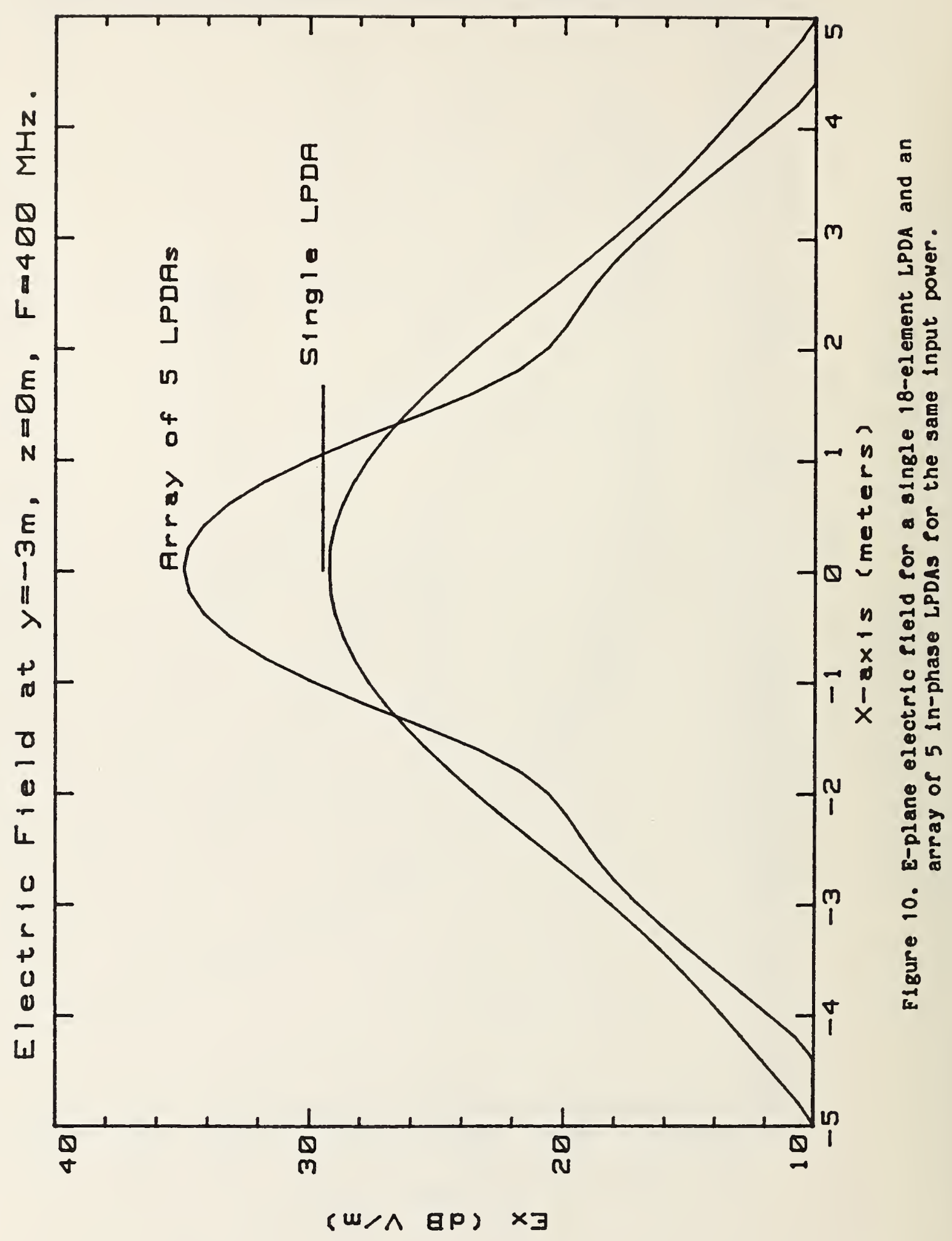




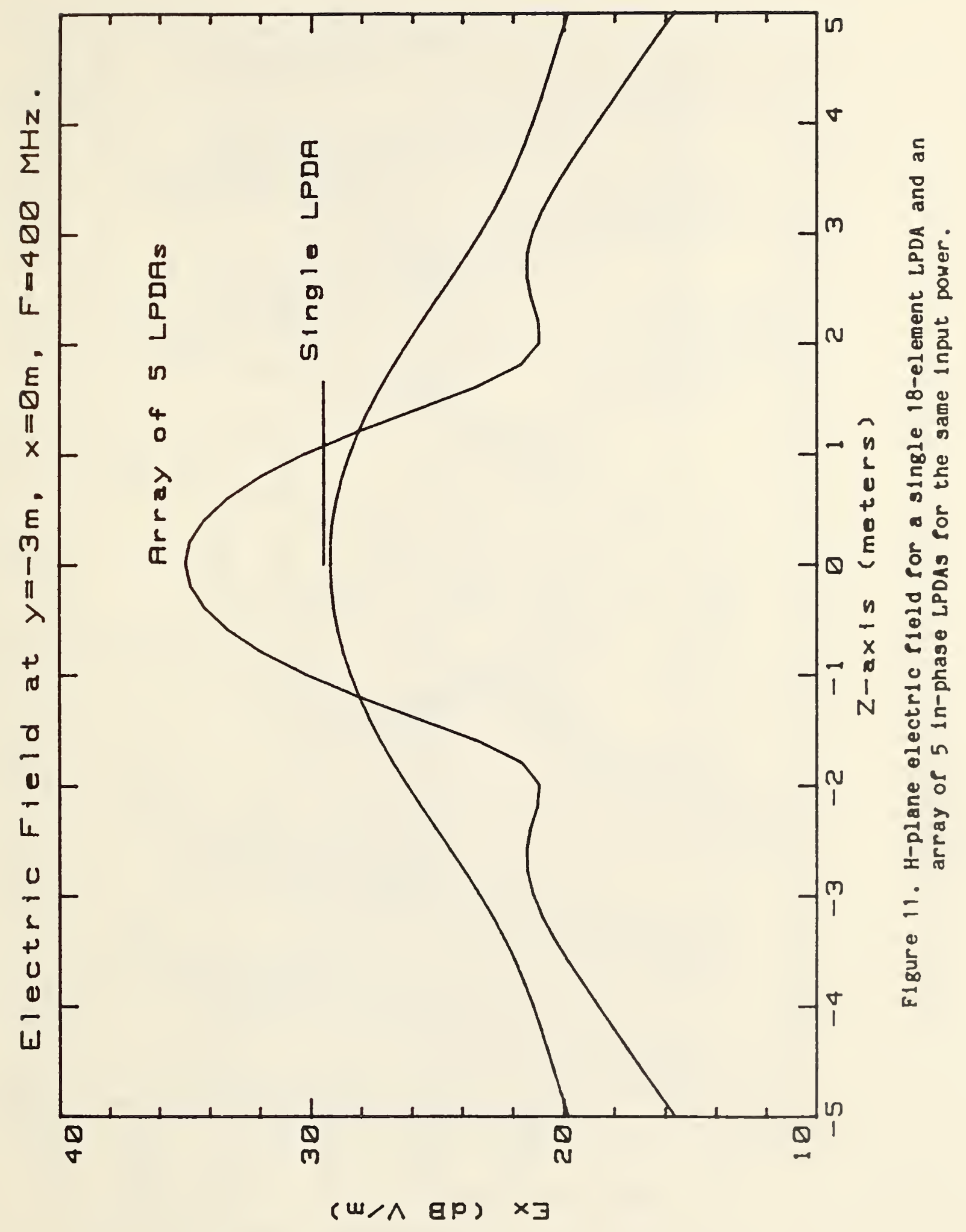




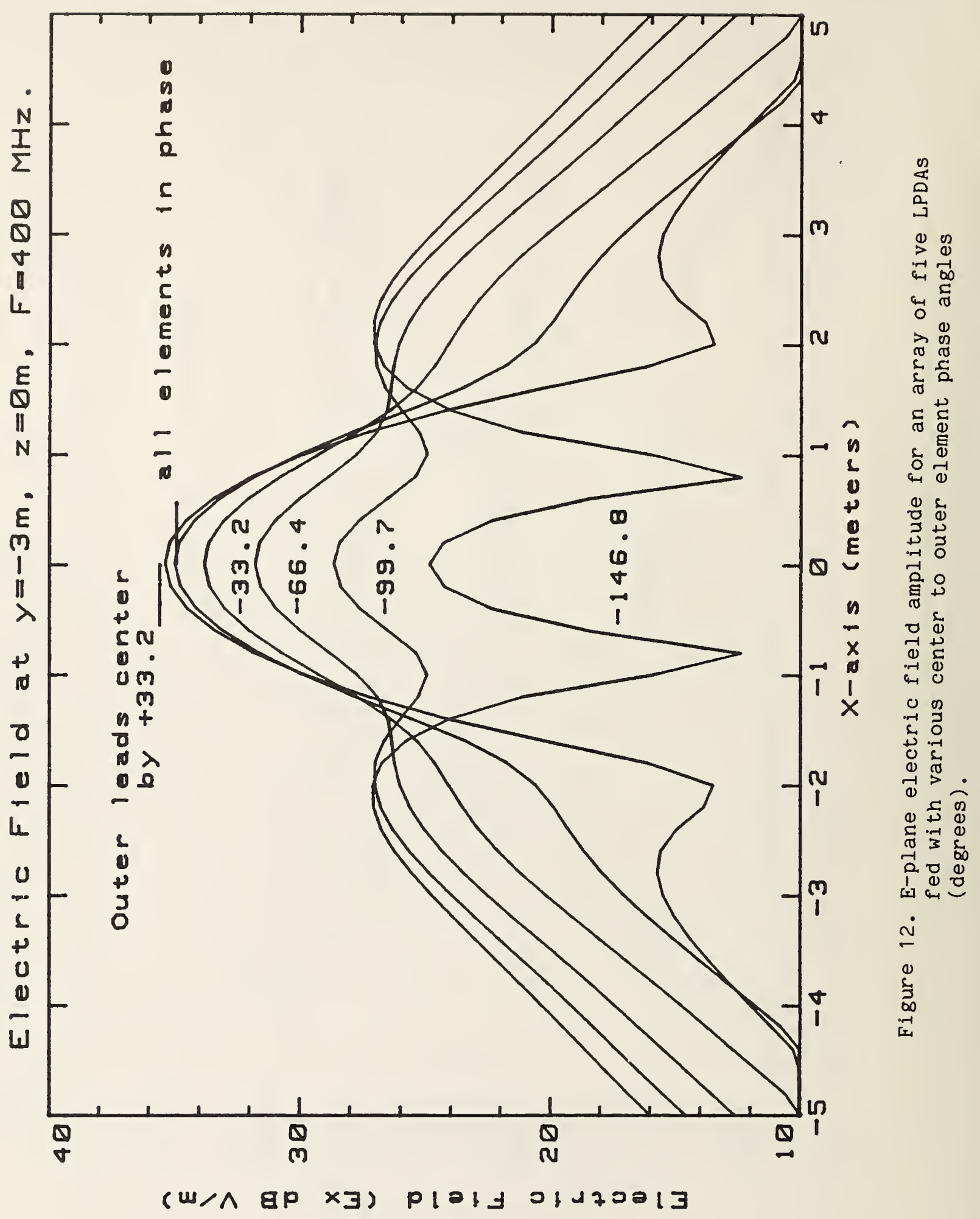




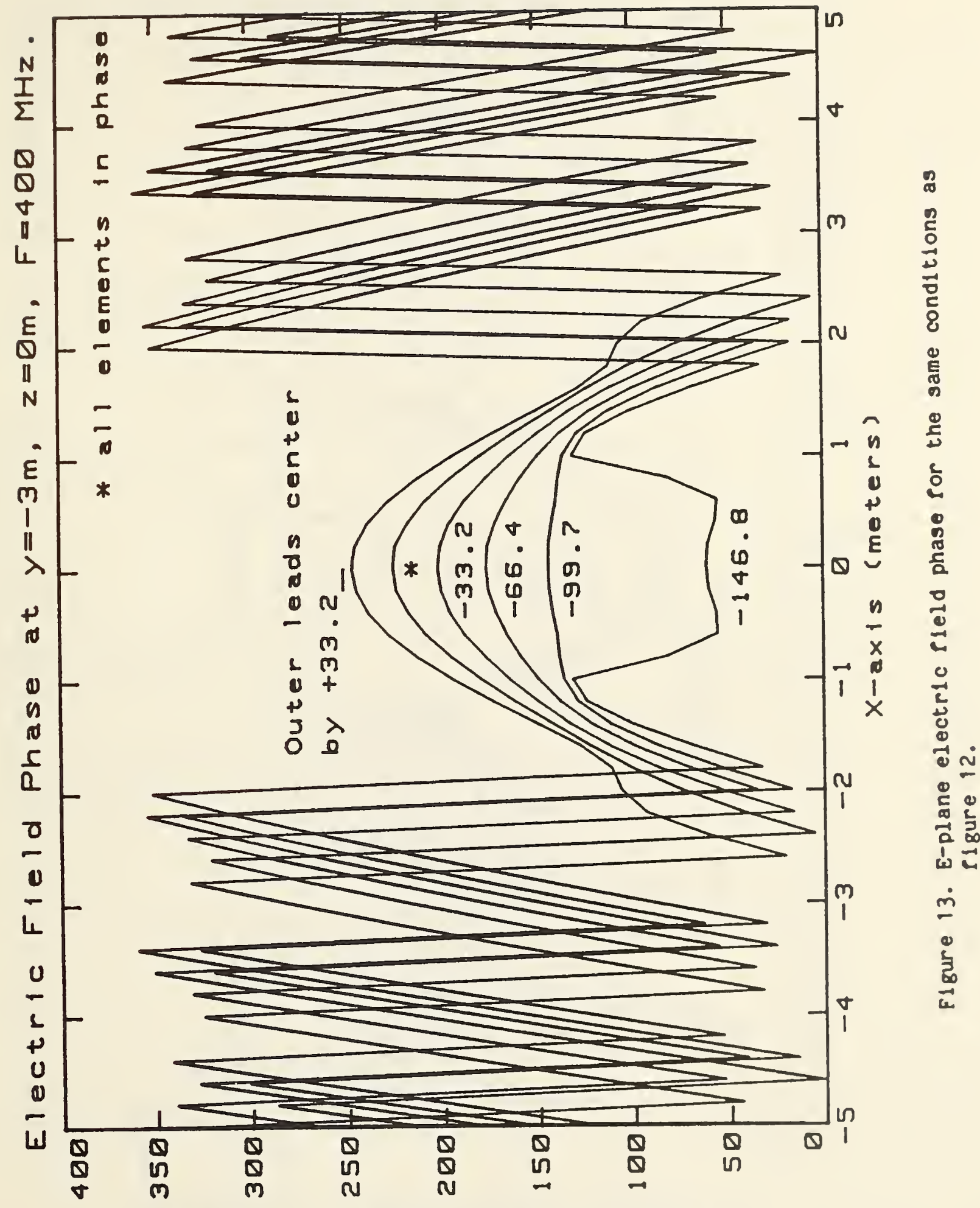

(soon6op) aseyd $p(01 t 51470013$ 


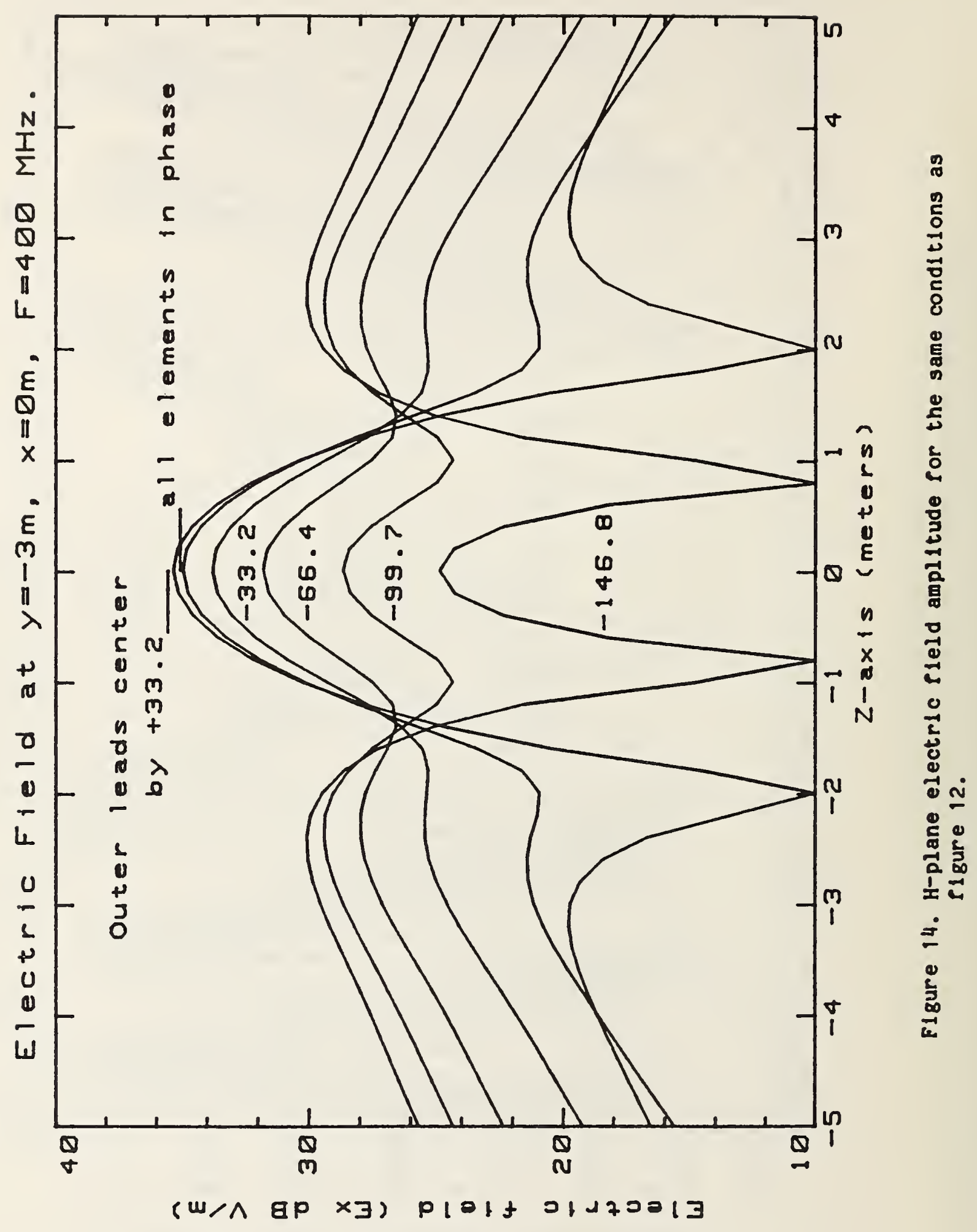




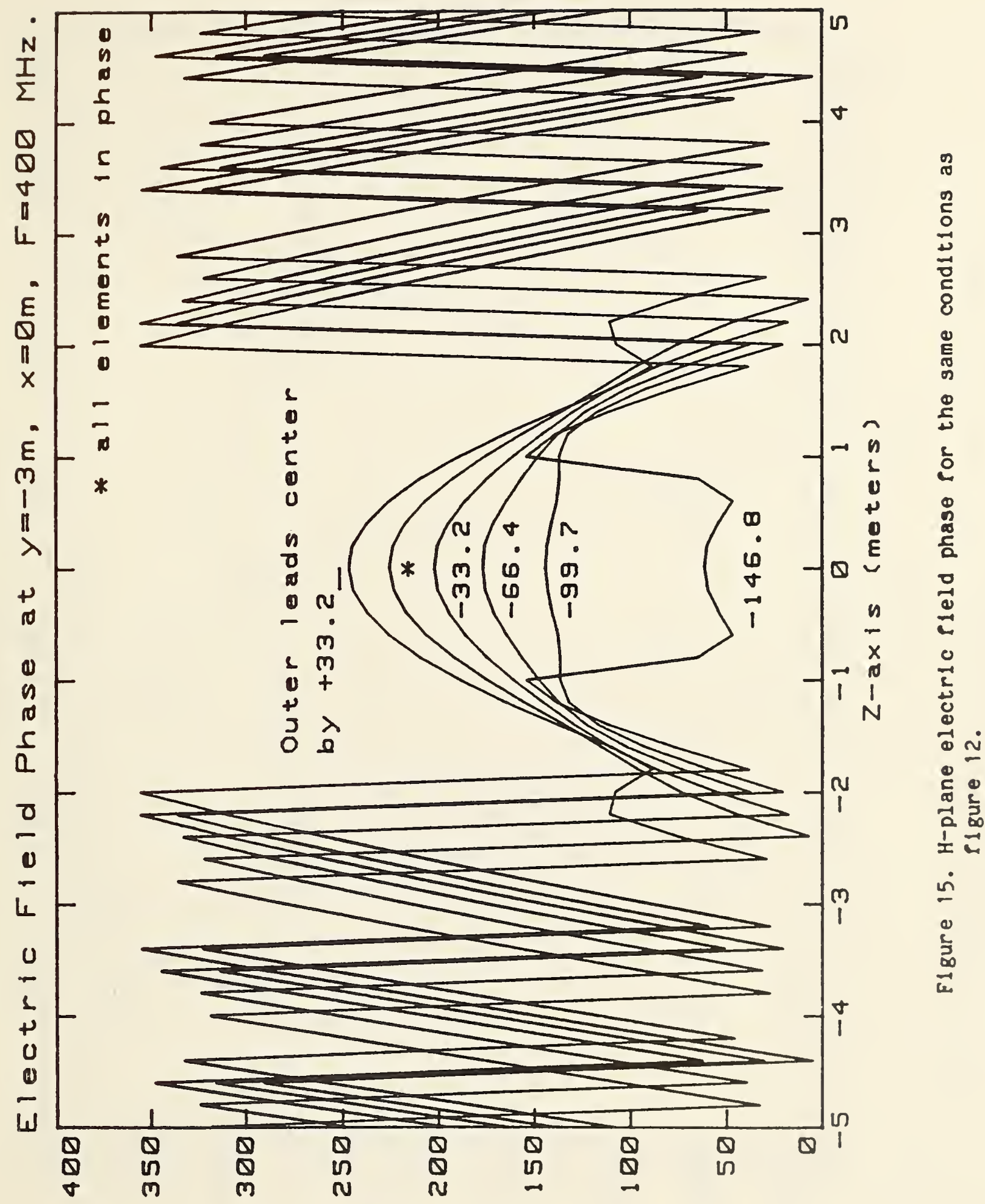

$(500 \lambda 60 p)$ oseyd $p 101 f 01+70013$ 


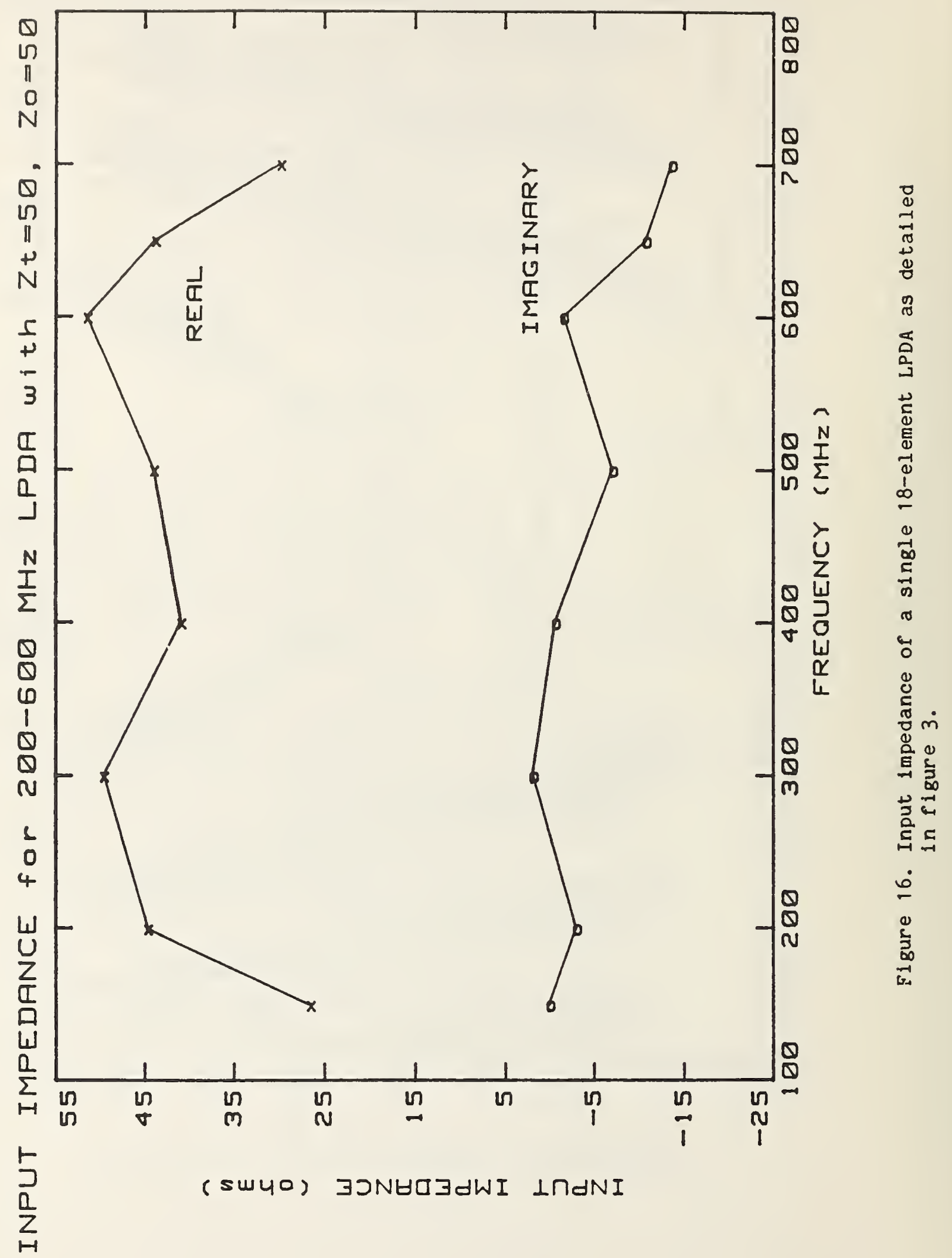




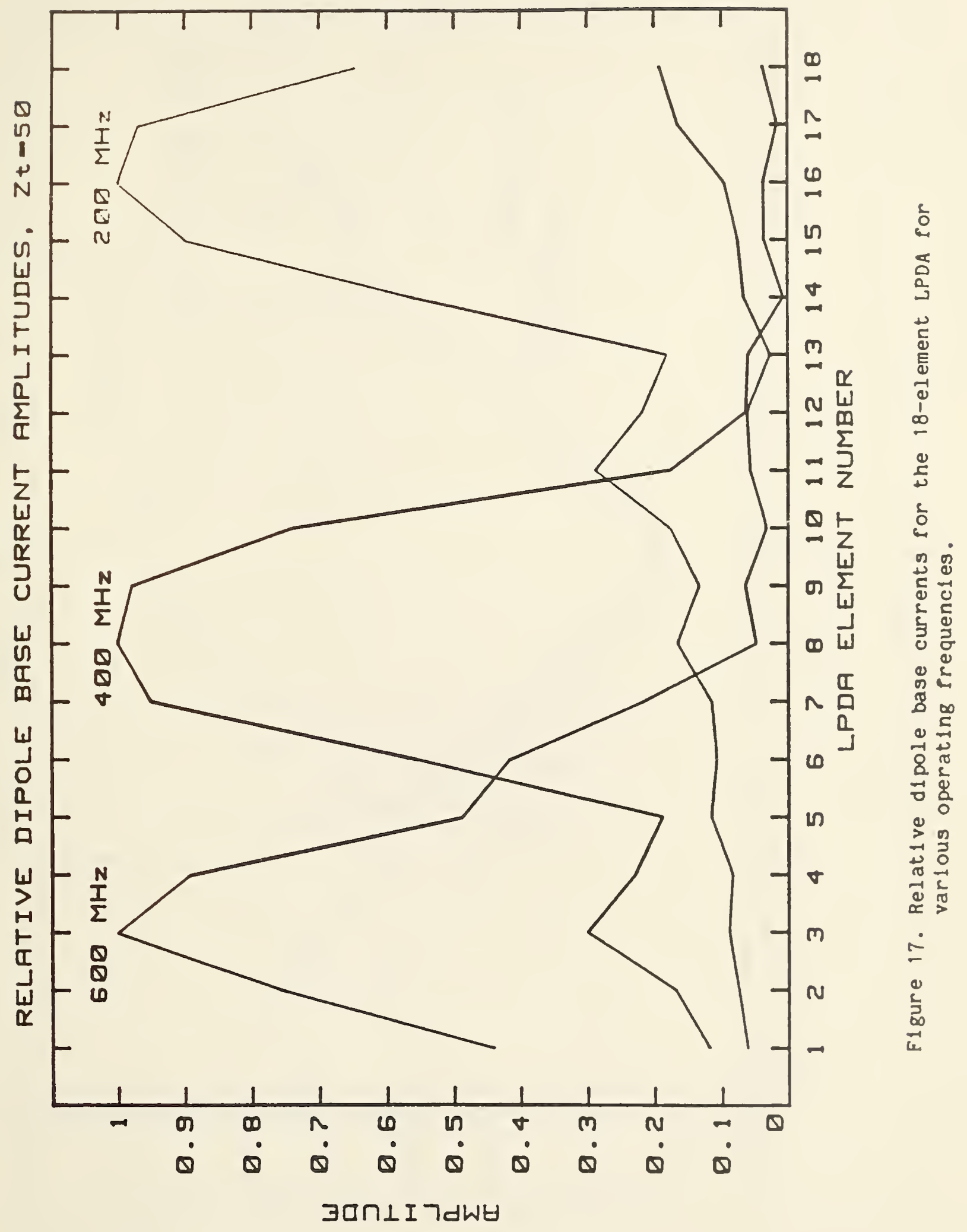




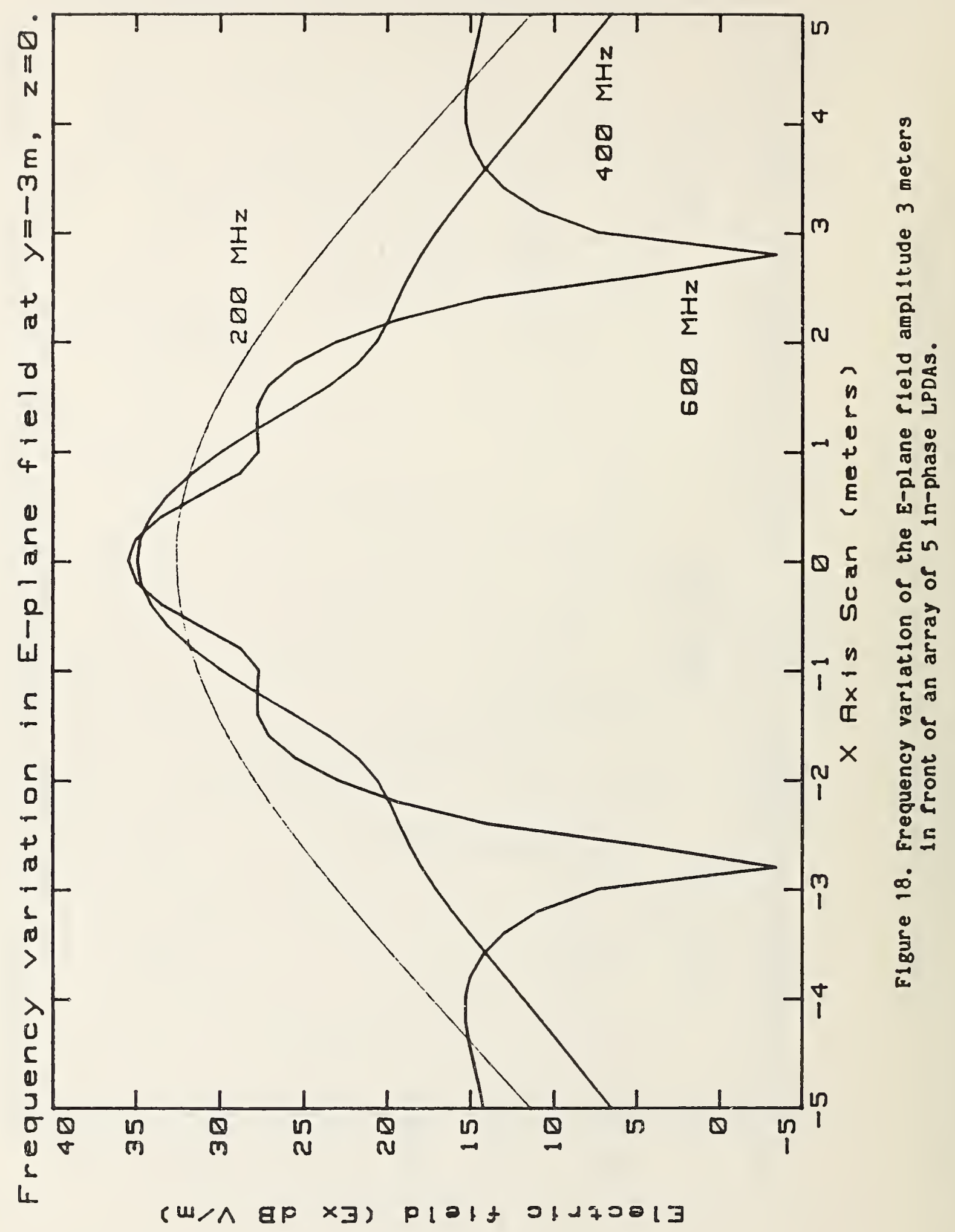




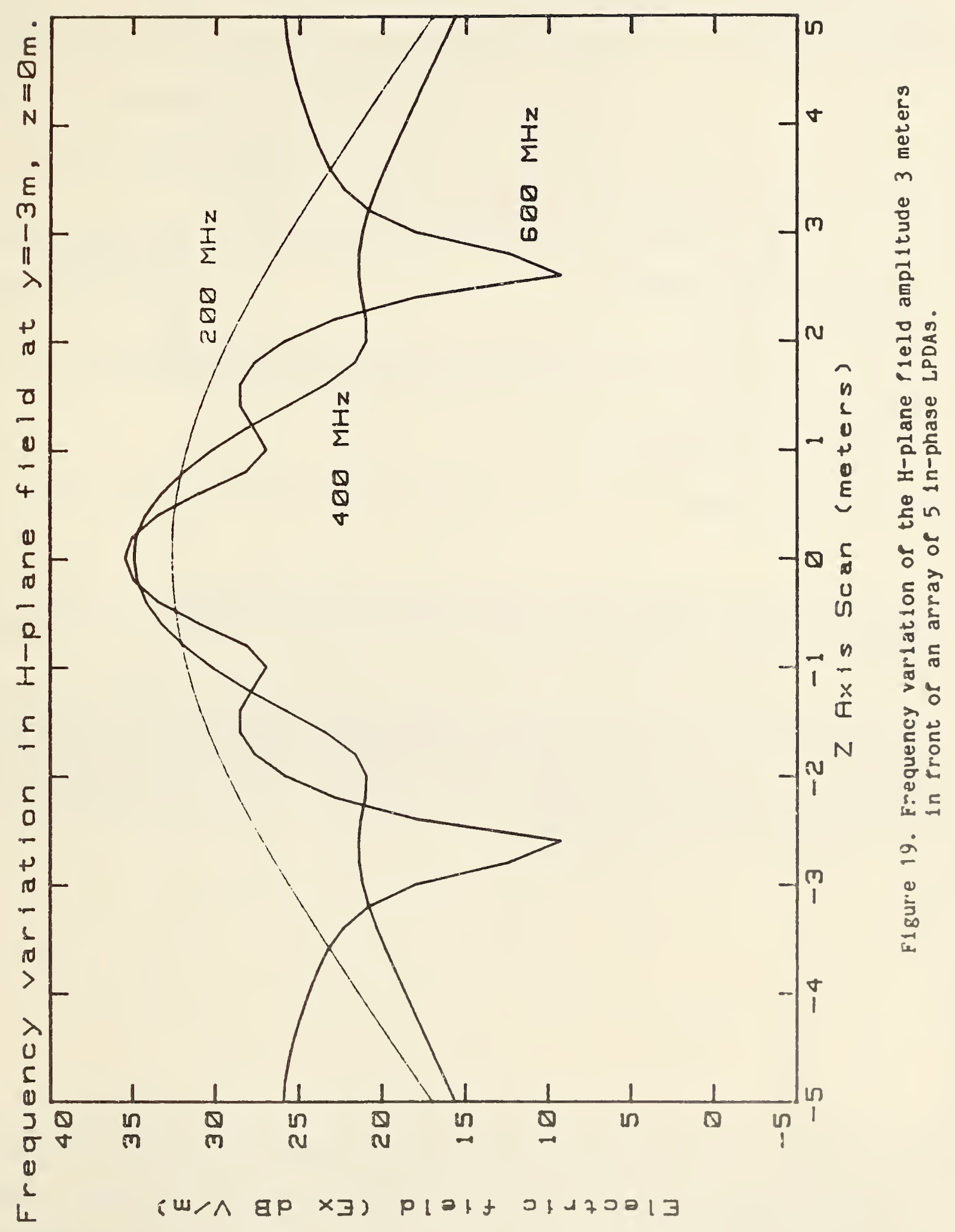


NBS-114A (REV. 2-80)

\begin{tabular}{|c|c|c|c|}
\hline $\begin{array}{c}\text { U.S. DEPT. OF COMM. } \\
\text { BIBLIOGRAPHIC DATA } \\
\text { SHEET (See instructions) }\end{array}$ & $\begin{array}{c}\text { 1. PUBLICATION OR } \\
\text { REPORT NO. }\end{array}$ & 2. Performing Organ. Report No. & 3. Publication Date \\
\hline
\end{tabular}

4. TITLE AND SUBTITLE

Analysis of an Array of Log-Periodic Dipole Antennas for Generating Test Fields

5. $\operatorname{AUTHOR}(S)$

Galen H. Koepke, David A. Hill, and Mark T. Ma

6. PERFORMING ORGANIZATION (If joint or other than NBS, see instructions)

7. Contract/Grant No.

NATIONAL BUREAU OF STANDARDS

DEPARTMENT OF COMMERCE

8. Type of Report \& Period Covered

WASHINGTON, D.C. 20234

9. STCHSOFHW ORGAIHZATIOH NAME AND COMPLETE ADDRESS (Street. CHY, State, ZIF)

Sponsored in part by

Naval Surface Weapons Center

Dahlgren, Virginia 22448

10. SUPPLEMENTARY NOTES

[] Document describes a computer program; SF-185, FIPS Software Summary, is attached.

11. ABSTRACT (A 200-word or less factual summary of most significant information. If document includes a significant bibliography or literature survey, mention it here)

An analysis of log-periodic dipole antennas was extended to study their use in arrays designed for electromagnetic susceptibility measurements. Parameters of an array of five log-periodic dipole antennas were calculated and in some cases compared to a single log-periodic dipole antenna. These parameters were used to evaluate the tradeoffs that exist in the design of an optimum transmitting antenna for susceptibility measurements.

12. KEY WORDS (Six to twelve entries; alphabetical order; capitalize only proper names; and separate key words by semicolons)

broadband antenna; electromagnetic susceptibility; log-periodic dipole antenna; near-field phase array

13. AVAILABILITY

X Unlimited

For Official Distribution. Do Not Release to NTIS

Order From Superintendent of Documents, U.S. Government Printing Office, Washington, D.C. 20402.

14. NO. OF

PRINTED PAGES

40

15. Price

X] Order From National Technical Information Service (NTIS), Springfield, VA. 22161 

